Proyecciones Journal of Mathematics

Vol. 36, No 2, pp. 195-208, June 2017.

Universidad Católica del Norte

Antofagasta - Chile

\title{
A study on prime arithmetic integer additive set-indexers of graphs
}

\author{
N. K. Sudev \\ Centre for Studies in Discrete Mathematics, India \\ and \\ K. A. Germina \\ Central University of Kerala, India \\ Received: October 2015. Accepted: March 2017
}

\begin{abstract}
Let $\mathbf{N}_{0}$ be the set of all non-negative integers and $\mathcal{P}\left(\mathbf{N}_{0}\right)$ be its power set. An integer additive set-indexer (IASI) is defined as an injective function $f: V(G) \rightarrow \mathcal{P}\left(\mathbf{N}_{0}\right)$ such that the induced function $f^{+}: E(G) \rightarrow \mathcal{P}\left(\mathbf{N}_{0}\right)$ defined by $f^{+}(u v)=f(u)+f(v)$ is also injective, where $\mathbf{N}_{0}$ is the set of all non-negative integers. A graph $G$ which admits an IASI is called an IASI graph. An IASI of a graph $G$ is said to be an arithmetic IASI if the elements of the set-labels of all vertices and edges of $G$ are in arithmetic progressions. In this paper, we discuss about a particular type of arithmetic IASI called prime arithmetic IASI.
\end{abstract}

Key words: Integer additive set-indexers, arithmetic integer additive set-indexers, prime arithmetic integer additive set-indexers.

AMS Subject Classification : 05C78 


\section{Introduction}

For all terms and definitions, not defined in this paper, we refer to [12] and for more about graph labeling, we refer to [8]. Unless mentioned otherwise, all graphs considered here are simple, finite and have no isolated vertices.

Let $\mathbf{N}_{0}$ denote the set of all non-negative integers. For all $A, B \subseteq \mathbf{N}_{0}$, the sum set of these sets, denoted by $A+B$, is defined by $A+B=\{a+b$ : $a \in A, b \in B\}$. If either $A$ or $B$ is infinite, then $A+B$ is also infinite. Hence, all sets mentioned in this paper are finite sets of non-negative integers. We denote the cardinality of a set $A$ by $|A|$.

An integer additive set-indexer (IASI, in short) is defined in [9] as an injective function $f: V(G) \rightarrow \mathcal{P}\left(\mathbf{N}_{0}\right)$ such that the induced function $f^{+}$: $E(G) \rightarrow \mathcal{P}\left(\mathbf{N}_{0}\right)$ defined by $f^{+}(u v)=f(u)+f(v)$ is also injective (see $[9,10,16])$. A graph $G$ which admits an IASI is called an IASI-graph.

An IASI is said to be $k$-uniform if $\left|f^{+}(e)\right|=k$ for all $e \in E(G)$. That is, a connected graph $G$ is said to have a $k$-uniform IASI if all of its edges have the same set-indexing number $k$.

The cardinality of the labeling set of an element (vertex or edge) of a graph $G$ is called the set-indexing number of that element. The vertex set $V$ of a graph $G$ is defined to be $l$-uniformly set-indexed, if all the vertices of $G$ have the same set-indexing number $l$. An element of $G$ whose set-indexing number 1 is called a mono-indexed element of $G$.

By the term an AP-set, we mean a set whose elements are in arithmetic progression. In this paper, we study the characteristics given graphs, where the set-labels of whose vertices and edges are AP-sets. Since the elements of the set-labels of all elements of $G$ are in arithmetic progression, all setlabels must contain at least three elements. The common difference of the set-label of an element $x$ of a graph $G$ is called the deterministic index of $x$ and is denoted by $\varpi(x)$. The integer ratio between the deterministic indices of the end vertices of an edge $e$ in $G$ is called the deterministic ratio of $e$.

Certain types of arithmetic integer additive set-indexers of given graphs have been introduced in [18] as follows.

Let $f: V(G) \rightarrow \mathcal{P}\left(\mathbf{N}_{0}\right)$ be an IASI on $G$. For any vertex $v$ of $G$, if $f(v)$ is an AP-set, then $f$ is called a vertex-arithmetic IASI of $G$. A graph that admits a vertex-arithmetic IASI is called a vertex-arithmetic IASI graph.

For an IASI $f$ of $G$, if $f^{+}(e)$ is an AP-set, for all $e \in E(G)$, then $f$ is called an edge-arithmetic IASI of $G$. A graph that admits an edgearithmetic IASI is called an edge-arithmetic IASI graph. An IASI is said 
to be an arithmetic integer additive set-indexer (arithmetic IASI, in short) if it is both vertex-arithmetic and edge-arithmetic. That is, an arithmetic IASI is an IASI $f$, under which the set-labels of all elements of a given graph $G$ are AP-sets. A graph that admits an arithmetic IASI is called an arithmetic IASI graph.

Certain studies on the characteristics and structural properties of arithmetic IASI-graphs have been done in $[18,19,20]$ and the following are some of the major results which are relevant in our present study.

Theorem 1.1. [18] A graph $G$ admits an arithmetic IASI if and only if for any two adjacent vertices in $G$, the deterministic index of one vertex is a positive integral multiple of the deterministic index of the other vertex and this positive integer is less than or equal to the set-indexing number of the latter.

Let $v_{i}$ and $v_{j}$ are two adjacent vertices of $G$, with deterministic indices $d_{i}$ and $d_{j}$ respectively with respect to an IASI $f$ of $G$. Then, by Theorem $1.1, f$ is an arithmetic IASI if and only if $d_{j}=k d_{i}$, where $k$ is a positive integer such that $1 \leq k \leq\left|f\left(v_{i}\right)\right|$ or equivalently, the deterministic ratio of the edge $u v$ is a positive integer less than or equal to the set-indexing number of the vertex having smaller deterministic index.

Theorem 1.2. [18] Let $G$ be a graph which admits an arithmetic IASI, say $f$ and let $d_{i}$ and $d_{j}$ be the deterministic indices of two adjacent vertices $v_{i}$ and $v_{j}$ in $G$. If $\left|f\left(v_{i}\right)\right| \geq\left|f\left(v_{j}\right)\right|$, then for some positive integer $1 \leq k \leq$ $\left|f\left(v_{i}\right)\right|$, the edge $v_{i} v_{j}$ has the set-indexing number $\left|f\left(v_{i}\right)\right|+k\left(\left|f\left(v_{j}\right)\right|-1\right)$.

In this paper, we introduce the notion of prime arithmetic IASI-graphs and study the properties and characteristics of them.

\section{Prime arithmetic IASI Graphs}

In this section, we first define a particular type of biarithmetic IASI of a given graph as follows.

Definition 2.1. A prime arithmetic integer additive set-indexer (prime arithmetic IASI) of a graph $G$ is an arithmetic IASI $f: V(G) \rightarrow \mathcal{P}\left(\mathbf{N}_{0}\right)$, where, for any two adjacent vertices in $G$ the deterministic index of one vertex is a prime integer multiple of the deterministic index of the other, where this prime integer is less than or equal to the set-indexing number of the second vertex. 
In other words, an arithmetic IASI $f$ is a prime arithmetic IASI on $G$ if for any two adjacent vertices $v_{i}$ and $v_{j}$ of $G$ with the deterministic indices $d_{i}$ and $d_{j}$ respectively where $d_{i} \leq d_{j}, d_{j}=p_{i} d_{i}$ where $p_{i}$ is a prime integer such that $1<p_{i} \leq\left|f\left(v_{i}\right)\right|$.

In the following discussions, we discuss the admissibility of prime arithmetic IASI by certain graphs. The following theorem discusses a necessary and sufficient condition for a given graph to admit a prime arithmetic IASI.

Theorem 2.2. A graph $G$ admits a prime arithmetic IASI if and only if it is bipartite.

Proof. Let $G$ be a bipartite graph with a bipartition $(X, Y)$. Label all the vertices in $X$ by distinct AP-sets having the same common difference $d$ and label all the vertices of $Y$ by distinct AP-sets having the same common difference $d^{\prime}$, where $d^{\prime}=p d$, where $p=\min _{v \in X}|f(v)|$ is a prime integer. This set-labeling is a prime-arithmetic IASI defined on $G$.

Conversely, assume that $G$ admits a prime arithmetic IASI, say $f$. Let $v$ be an arbitrary vertex of $G$. Let $V_{1}$ denote the set of all vertices that are adjacent to $v$. Since $G$ is a prime arithmetic IASI graph, for any vertex $v^{\prime}$ in $V_{1}$, either $\varpi\left(v^{\prime}\right)=p_{i} \varpi(v) ; p_{i} \leq|f(v)|$ (or $\varpi(v)=p_{j} \varpi(v) ; p_{i} \leq\left|f\left(v^{\prime}\right)\right|$ ), where $p_{i}$ (or $p_{j}$ ) is a prime integer. Now, let $V_{2}$ be the neighbouring set of $V_{1}$. Since, $G$ admits a prime arithmetic IASI, no vertices in $V_{2}$ can be adjacent to $v$. Let $V_{3}$ be the neighbouring set of the vertices of $V_{2}$ and as explained above no vertices of $V_{1}$ will be adjacent to the vertices in $V_{3}$. Proceeding like this in finite number of times, say $m$, we cover all the vertices of $G$. Let $X=\bigcup_{i=0}^{m} V_{2 i}$ and $Y=\bigcup_{i=0}^{m} V_{2 i+1}$. Clearly, $(X, Y)$ is a bipartition on $G$.

The following are some illustrations of the graphs which admit prime arithmetic IASIs.

Remark 2.3. Due to Theorem 2.2, the paths, trees, even cycles and all acyclic graphs admit prime arithmetic IASIs.

Illustration 2.4. The subdivision graph of any non-trivial graph $G$ admits a prime arithmetic IASI graph.

A subdivision graph, denoted by $G^{*}$, is the graph obtained by introducing a vertex to every edge of $G$. Then, $G^{*}$ is a bipartite graph. Hence, by Theorem 2.2, $G^{*}$ admits a prime arithmetic IASI. 
Illustration 2.5. Every median graph admits a prime arithmetic IASI.

A median graph $G$ is an undirected graph in which every three vertices $u, v$ and $w$ have a unique vertex $x$ that belongs to shortest paths between each pair of $u, v$,and $w$. Clearly, $G$ is a bipartite graph and hence by Theorem 2.2, $G$ admits a prime-arithmetic IASI.

Illustration 2.6. Every hypercube graph $Q_{n}$ and partial cube graph admit a prime arithmetic IASI.

Every hypercube graph $Q_{n}$ is a Cartesian product of two bipartite graphs and hence it is a bipartite graph. Then, by Theorem $2.2, Q_{n}$ admits a primearithmetic IASI. A partial cube is a graph that is an isometric subgraph of a hypercube. Since it preserves distances, it is also a bipartite graph and hence admits a prime arithmetic IASI.

\section{Dispensing Number of Certain Graph Classes}

By Theorem 2.2, a non-bipartite graph does not admit a prime arithmetic IASI. That is, some edges of a non-bipartite graph have non-prime deterministic ratio. Then, we define the following notion.

Definition 3.1. The minimum possible number of edges in a graph $G$ that do not have a prime deterministic ratio is called the dispensing number of $G$ and is denoted by $\vartheta(G)$.

In other words, the dispensing number of a graph $G$ is the minimum number of edges to be removed from $G$ so that it admits a prime arithmetic IASI. Hence, we have

Theorem 3.2. If $b(G)$ is the number of edges in a maximal bipartite subgraph of a graph $G$, then $\vartheta(G)=|E(G)|-b(G)$.

Proof. Let $G$ be a non-bipartite graph which has $n$ vertices and $m$ edges. Let $f$ be an arithmetic IASI defined on $G$ which labels the vertices of $G$ in such a way that maximum number of edges have a prime deterministic ratio. This can be done by assigning distinct AP-sets to the vertices of $G$ in such a way that the deterministic index of a vertex (other than the vertex having the smallest deterministic index) is a prime multiple of the common difference of the set-label of some other vertex in $G$. Let $E_{p}$ be the set of 
all edges in $G$ which have a prime deterministic ratio in $G$ with respect to $f$. Then, clearly the induced subgraph $\left\langle E_{p}\right\rangle$ of $G$ is a maximal bipartite subgraph of $G$. The edges in $G-\left\langle E_{p}\right\rangle$ do not have a prime deterministic ratio. Therefore, $\vartheta(G)=\left|G-\left\langle E_{p}\right\rangle\right|=|E(G)|-\left|\left\langle E_{p}\right\rangle\right|=|E(G)|-b(G)$.

Invoking Theorem 3.2, we investigate the sparing number about the dispensing number of certain graph classes.

Proposition 3.3. The dispensing number of an odd cycle is 1 .

Proof. Let $C_{n}$ be an odd cycle. Since no bipartite graphs contain odd cycles, $C_{n}$ is not a bipartite graphs. Let $e$ be any edge of $C_{n}$. Then, $C_{n}-e$ is a path on $n$ vertices, which is a bipartite graph. Therefore, for odd $n$, $\vartheta\left(C_{n}\right)=1$.

The union of two graphs $G_{1}$ and $G_{2}$, denoted by $G_{1} \cup G_{2}$, is the graph whose vertex set is $V\left(G_{1}\right) \cup V\left(G_{2}\right)$ and the edge set $E\left(G_{1}\right) \cup E\left(G_{2}\right)$. If two graphs $G_{1}$ and $G_{2}$ have no common elements, then their union is said to be the disjoint union. The following theorem estimates the dispensing number of the union of two graphs.

Theorem 3.4. Let $G_{1}$ and $G_{2}$ be two given IASI-graphs. Then, $\vartheta\left(G_{1} \cup\right.$ $\left.G_{2}\right)=\vartheta\left(G_{1}\right)+\vartheta\left(G_{2}\right)-\vartheta\left(G_{1} \cap G_{2}\right)$. In particular, if $G_{1}$ and $G_{2}$ are two edge-disjoint graphs, then $\vartheta\left(G_{1} \cup G_{2}\right)=\vartheta\left(G_{1}\right)+\vartheta\left(G_{2}\right)$.

Proof. Let $G_{1}$ and $G_{2}$ admit arithmetic IASIs, say $f_{1}$ and $f_{2}$ respectively such that $f_{1}\left(v_{i}\right) \neq f_{2}\left(w_{j}\right)$ for any two distinct vertices $v_{i} \in V\left(G_{1}\right)$ and $w_{j} \in V\left(G_{2}\right)$. Define a function $f: V\left(G_{1} \cup G_{2}\right) \rightarrow \mathcal{P}\left(\mathbf{N}_{0}\right)$ as follows.

$$
f(v)= \begin{cases}f_{1}(v) & \text { if } v \in V\left(G_{1}\right) \\ f_{2}(v) & \text { if } v \in V\left(G_{2}\right) .\end{cases}
$$

If $G_{1}$ and $G_{2}$ are edge-disjoint, no edge in $G_{1}$ has the same set-label of a vertex in $G_{2}$. Hence $f$ is injective. Therefore, $\vartheta\left(G_{1} \cup G_{2}\right)=\vartheta\left(G_{1}\right)+\vartheta\left(G_{2}\right)$.

If $G_{1}$ and $G_{2}$ have some edges in common, then $G_{1} \cap G_{2}$ is the graph induced by these edges. Then, $f_{1}=f_{2}$ for all the vertices in $G_{1} \cap G_{2}$. Therefore, the graphs $G_{1}-G_{1} \cap G_{2}, G_{2}-G_{1} \cap G_{2}$ and $G_{1} \cap G_{2}$ are disjoint graphs whose union is the graph $G_{1} \cup G_{2}$. Hence, $G_{1} \cup G_{2}=\left(G_{1}-G_{1} \cap G_{2}\right) \cup$ $\left(G_{2}-G_{1} \cap G_{2}\right) \cup\left(G_{1} \cap G_{2}\right)$. Then, by the above argument, $\vartheta\left(G_{1} \cup G_{2}\right)=$ $\vartheta\left(G_{1}-G_{1} \cap G_{2}\right)+\vartheta\left(G_{2}-G_{1} \cap G_{2}\right)+\vartheta\left(G_{1} \cap G_{2}\right)=\vartheta\left(G_{1}\right)+\vartheta\left(G_{2}\right)-\vartheta\left(G_{1} \cap G_{2}\right)$. This completes the proof. 
Invoking Theorem 3.4, we establish the following theorem on the dispensing number of Eulerian graphs.

Theorem 3.5. The dispensing number of an Eulerian graph $G$ is the number of odd cycles in $G$.

Proof. Let $G$ be an Eulerian graph. Then, $G$ can be decomposed into a finite number of edge disjoint cycles. Let $C_{n_{1}}, C_{n_{2}}, C_{n_{3}}, \ldots, C_{n_{l}}$ be the edge disjoint cycles in $G$ such that $G=\cup_{i=1}^{l} C_{n_{i}}$. Without loss of generality let $C_{n_{1}}, C_{n_{2}}, C_{n_{3}} \ldots C_{n_{r}}$ are odd cycles $C_{n_{r+1}}, C_{n_{r+2}}, \ldots, C_{n_{l}}$ are even cycles. By Theorem 2.2, the dispensing number of even cycles is 0 and by Proposition 3.3, the dispensing number of odd cycles is 1 . Since The cycles in $G$ are edge disjoint, all odd cycles in $G$ has one edge that has no prime deterministic ratio. Therefore, by Theorem 3.4, the dispensing number of $G$ is equal to the number of odd cycles in $G$.

A cactus $G$ is a connected graph in which any two simple cycles have at most one vertex in common. That is, every edge in a cactus belongs to at most one simple cycle. The cycles in a cactus are edge disjoint. Hence, we have

Theorem 3.6. The dispensing number of a cactus $G$ is the number of odd cycles in $G$.

Proof. Let $G$ be a cactus. Let $C_{n_{1}}, C_{n_{2}}, C_{n_{3}}, \ldots, C_{n_{l}}$ be cycles in $G$. All these cycles are edge disjoint. Without loss of generality let

$C_{n_{1}}, C_{n_{2}}, C_{n_{3}} \ldots C_{n_{r}}$ are odd cycles $C_{n_{r+1}}, C_{n_{r+2}}, \ldots, C_{n_{l}}$ are even cycles. By Theorem 2.2, the dispensing number of even cycles is 0 and by Proposition 3.3, the dispensing number of odd cycles is 1 . Then, all odd cycles in $G$ has one edge that has no prime deterministic ratio. Therefore, by Theorem 3.4, the dispensing number of $G$ is equal to the number of odd cycles in $G$.

An interesting graph class we wish to study in this context is the class of wheel graphs. A wheel graph, denoted by $W_{n+1}$ is the graph obtained by joining every vertex of a cycle $C_{n}$ to an external vertex. The following result discusses the dispensing number of wheel graphs.

Proposition 3.7. The dispensing number of a wheel graph $W_{n+1}$ is given by $\left\lceil\frac{n}{2}\right\rceil$. 
Proof. Let $W_{n+1}=C_{n}+K_{1}$. Let $v$ be the central vertex and $v_{1}, v_{2}, \ldots, v_{n}$ be the vertices of the cycle $C_{n}$ in $W_{n+1}$. Then, any two adjacent vertices in $C_{n}$, together with the central vertex $v$ induce a triangle in $W_{n+1}$ and hence $W_{n+1}$ is not a bipartite graph. Here, we need to consider the following two cases.

(i) Let $n$ be even. Then, $C_{n}$ is bipartite. Also, there are $n$ triangles in $W_{n+1}$ such that any two neighbouring triangles have a common edge. We can group these triangles into $\frac{n}{2}$ distinct pairs of neighbouring triangles having exactly one common edge. On removing this common edge, each pair of these triangles reduces to a cycle $C_{4}$, which is bipartite. Hence, removal of the common edges from all pairs of triangles, the graph reduces to the union of even cycles. Hence, the reduced graph is a (maximal) bipartite subgraph of $W_{n+1}$. In this case, the minimum number of edges removed from the wheel graph $W_{n+1}$ is $\frac{n}{2}$.

(ii) Let $n$ be odd. Then, $C_{n}$ is not bipartite and one edge of $C_{n}$ is to be removed so that it becomes bipartite. Here, also we have $n$ triangles in $W_{n+1}$ and as mentioned in the previous case of which, we can group these triangles into $\left\lfloor\frac{n}{2}\right\rfloor$ distinct pairs of neighbouring triangles which have exactly one common edge and one triangle will remain unpaired. First, remove the edge common to this unpaired triangle and the cycle $C_{n}$. so that both become bipartite. Now, as mentioned in the previous case, remove the common edges from all paired triangles. Now, the wheel graph is reduced to the union of even cycles and hence the reduced graph is bipartite. In this case, the minimum number of edges removed from the wheel graph $W_{n+1}$ is $1+\left\lfloor\frac{n}{2}\right\rfloor$.

Combining both the above cases, we have $\vartheta\left(W_{n+1}\right)=\left\lceil\frac{n}{2}\right\rceil$.

This completes the proof.

In the next theorem, we discuss the dispensing number of the join of two graphs.

Theorem 3.8. Let $G_{1}\left(V_{1}, E_{1}\right)$ and $G_{2}\left(V_{2}, E_{2}\right)$ be two non-empty graphs. The dispensing number of $G_{1}+G_{2}$ is $\vartheta\left(G_{1}+G_{2}\right)=\min \left\{\left|E_{1}\right|+\vartheta\left(G_{2}\right),\left|E_{2}\right|+\right.$ $\left.\vartheta\left(G_{1}\right)\right\}$.

Proof. Let $G=G_{1}+G_{2}$. Then, any pair of adjacent vertices in $G_{1}$ induces a triangle in $G$ together with any vertex in $G_{2}$. Similarly, any pair 
of adjacent vertices in $G_{2}$ induces a triangle in $G$ together with any vertex in $G_{1}$. Hence, the edges in either $G_{1}$ or $G_{2}$ must be completely removed as the first step to construct a bipartite subgraph of $G$.

If we remove all edges of $G_{1}$, then additionally $\vartheta\left(G_{2}\right)$ properly chosen edges are to be removed from $G_{2}$ also so that $G_{2}$ is also reduced to a bipartite graph. The resultant graph will be a bipartite graph and the number of edges removed here is $\left|E_{1}\right|+\vartheta\left(G_{2}\right)$.

If we are removing all edges of $G_{2}$, then additionally $\vartheta\left(G_{1}\right)$ edges are also to be removed from $G_{1}$ also so that $G_{1}$ is also reduced to a bipartite graph. Then, the resultant graph will be a bipartite graph and the number of edges removed here is $\left|E_{2}\right|+\vartheta\left(G_{1}\right)$.

Therefore, the dispensing number of $G_{1}+G_{2}$ is $\min \left\{\left|E_{1}\right|+\vartheta\left(G_{2}\right),\left|E_{2}\right|+\right.$ $\left.\vartheta\left(G_{1}\right)\right\}$.

Theorem 3.9. Let $G_{1}\left(V_{1}, E_{1}\right)$ and $G_{2}\left(V_{2}, E_{2}\right)$ be two non-empty graphs. The dispensing number of their Cartesian product $G_{1} \square G_{2}$ is $\vartheta\left(G_{1} \square G_{2}\right)=$ $\left|V_{1}\right| \vartheta\left(G_{2}\right)+\left|V_{2}\right| \vartheta\left(G_{1}\right)$.

Proof. Let $G=G_{1} \square G_{2}$. It is to be noted that there are $\left|V_{1}\right|$ copies of $G_{2}$ and $\left|V_{2}\right|$ copies of $G_{1}$ in $G_{1} \square G_{2}$, where the corresponding vertices of two copies of $G_{1}$ are connected according to the adjacency of the corresponding vertices in $G_{2}$ and the corresponding vertices of two copies of $G_{2}$ are connected according to the adjacency of the corresponding vertices in $G_{1}$. Then, we can see that $\vartheta\left(G_{1}\right)$ edges are to be removed from each copy of $G_{1}$ so that each of them becomes bipartite. Similarly, $\vartheta\left(G_{2}\right)$ edges are to be removed from each copy of $G_{2}$ so that each of them becomes bipartite. Hence, $V_{1}\left|\vartheta\left(G_{2}\right)+\right| V_{2} \mid \vartheta\left(G_{1}\right)$ copies are to be removed from $G$ so that it becomes bipartite. Therefore, $\vartheta\left(G_{1} \square G_{2}\right)=\left|V_{1}\right| \vartheta\left(G_{2}\right)+\left|V_{2}\right| \vartheta\left(G_{1}\right)$.

Theorem 3.10. Let $G_{1}\left(V_{1}, E_{1}\right)$ and $G_{2}\left(V_{2}, E_{2}\right)$ be two non-empty graphs. The dispensing number of their corona product $G_{1} \odot G_{2}$ is $\vartheta\left(G_{1} \odot G_{2}\right)=$ $\vartheta\left(G_{1}\right)+\left|V_{1}\right|\left|E_{2}\right|$.

Proof. It is to be noted that every edge of every copy of $G_{2}$ is a part of a triangle in $G_{1} \odot G_{2}$, induced by its end vertices and the corresponding vertex in $G_{1}$. Therefore, all edges of all copies of $G_{2}$ must be removed as the first step to reduce $G_{1} \odot G_{2}$ bipartite. Also, $\vartheta\left(G_{1}\right)$ edges are to be removed from $G_{1}$ so that its reduced graph also becomes bipartite. Therefore, a total of $\vartheta\left(G_{1}\right)+\left|V_{1}\right|\left|E_{2}\right|$ edges are to be removed from $G_{1} \odot G_{2}$ so that becomes bipartite. Therefore, $\vartheta\left(G_{1} \odot G_{2}\right)=\vartheta\left(G_{1}\right)+\left|V_{1}\right|\left|E_{2}\right|$. 
In view of Theorem 3.2, we discuss the dispensing number of complete graphs $K_{n}$ in the following theorem.

Theorem 3.11. The dispensing number of a complete graph $K_{n}$ is

$$
\vartheta\left(K_{n}\right)= \begin{cases}\frac{1}{4} n(n-2) & \text { if } n \text { is even } \\ \frac{1}{4}(n-1)^{2} & \text { if } n \text { is odd. }\end{cases}
$$

Proof. The maximal bipartite subgraph of $K_{n}$ is a complete bipartite graph $K_{a, b}$, where $a+b=n$. The number of edges in $K_{n}-K_{a, b}$ is $\frac{1}{2}(a+$ $b)(a+b-1)-a b=\frac{1}{2}\left(a^{2}+b^{2}-a-b\right)=\frac{1}{2}[a(a-1)+b(b-1)]$. Then, we have the following cases.

Case-1: Let $n$ be an even integer. Then, the number of edges in $K_{a, b}$ is maximum if $a=b=\frac{n}{2}$. Hence, by Theorem 2.2, the dispensing number of $K_{n}$ is $\frac{1}{2}\left[\frac{n}{2}\left(\frac{n}{2}-1\right)+\frac{n}{2}\left(\frac{n}{2}-1\right)\right]=\frac{1}{4} n(n-2)$.

Case-2: Let $n$ be an odd integer. Then, the number of edges in $K_{a, b}$ is maximum if $a=\left\lfloor\frac{n}{2}\right\rfloor=\frac{n+1}{2}$ and $b=\left\lceil\frac{n}{2}\right\rceil=\frac{n+1}{2}$ for odd $n$. Then, by Theorem 2.2, the dispensing number of $K_{n}$ is $\frac{1}{2}\left[\frac{n-1}{2}\left(\frac{n-1}{2}-1\right)+\frac{n+1}{2}\left(\frac{n+1}{2}-\right.\right.$ $1)]=\frac{1}{4}(n-1)^{2}$. This completes the proof.

Another interesting graph whose vertex set has two partitions is a split graph. A split graph is a graph whose vertex has two partitions of which one is an independent set, say $S$ and the subgraph induced by the other is a block, say $K_{r}$. A split graph is said to be a complete split graph if every vertex of the independent set $S$ is adjacent to all vertices of the block $K_{r}$.

Invoking Theorem 3.11, we estimate the dispensing number of a split graph and complete split graph in the following theorem.

Theorem 3.12. Let $G$ be a split graph with a block $K_{r}$ and an independent set $S$, where $K_{r} \cup\langle S\rangle$. Then,

$$
\vartheta(G)= \begin{cases}\frac{1}{4} r(r-2) & \text { if } r \text { is even } \\ \frac{1}{4}(r-1)^{2} & \text { if } r \text { is odd. }\end{cases}
$$

Proof. Since $S$ is an independent set in $G$, the maximal bipartite subgraph of $G$ is obtained by eliminating required number of edges from the block $K_{r}$ only. Since $K_{r}$ is a complete graph in $G$, by Theorem 3.11, the minimum number of edges to be removed from $K_{r}$ so that it becomes a bipartite graph is $\frac{1}{4} r(r-2)$ if $r$ even and $\frac{1}{4}(r-1)^{2}$ if $r$ is even. Therefore, 


$$
\vartheta(G)= \begin{cases}\frac{1}{4} r(r-2) & \text { if } r \text { is even } \\ \frac{1}{4}(r-1)^{2} & \text { if } r \text { is odd. }\end{cases}
$$

Another graph class similar to cactus graphs is a block graph (or a clique tree) which is defined as an undirected graph in which every biconnected component (block) is a clique. The following theorem estimates the dispensing number of a block graph

Theorem 3.13. The dispensing number of a block graph $G$ is the sum of the dispensing numbers of the cliques in $G$.

Proof. Let $K_{n_{1}}, K_{n_{2}}, K_{n_{3}}, \ldots K_{n_{l}}$ be the cliques in $G$. Since $G$ is a block graph, then any two cliques in it hs at most one common vertex. That is, all cliques in $G$ are edge disjoint. then, by Theorem 3.4, $\vartheta(G)=\vartheta\left(\cup_{i=1}^{l} K_{n_{i}}\right)=$ $\sum_{i=1}^{l} \vartheta\left(K_{n_{i}}\right)$.

\section{Conclusion}

In this paper, we have discussed some characteristics of graphs which admit a certain types of IASIs called prime arithmetic IASI. We have established some conditions for some graph classes to admit this types of arithmetic IASIs and have explained certain properties and characteristics of prime arithmetic IASI-graphs. Some of the problems in this area we have identified are the following.

A cactus is a subclass of outer planar graphs. An outer planar graph is a planar graph all of whose vertices belong to the exterior unbounded plane of the graph. In an outer planar, two simple cycles can have at most one edge in common.

Problem 1. Find the dispensing number of outer planar graphs.

Problem 2. Determine the dispensing number of other graph operations such as graph ringsums, graph complements etc.

Problem 3. The problem of finding the dispensing number of other graph products like strong product, direct product, lexicographic product, rooted product etc.

Problem 4. The problem of finding the dispensing number of various graph classes such as bisplit graphs, sun graphs, sunlet graphs etc. 
Problems related to the characterisation of different arithmetic IASIgraphs are still open. The IASIs under which the vertices of a given graph are labeled by different standard sequences of non negative integers, are also worth studying. The problems of establishing the necessary and sufficient conditions for various graphs and graph classes to admit certain IASIs still

remain unsettled. All these facts highlight a wide scope for further studies in this area.

\section{References}

[1] B. D. Acharya, Arithmetic graphs, J. Graph Theory, 14(3)(1990), pp. 275-299, (1990).

[2] B. D. Acharya, K. A. Germina and T. M. K. Anandavally, Some new perspective on arithmetic graphs in Labeling of Discrete Structures and Applications, (Eds.: B. D. Acharya, S. Arumugam and A. Rosa), Narosa Pub. House, New Delhi, pp. 41-46, (2008).

[3] T. M. Apostol, Introduction to analytic number theory, SpringerVerlag, New York, (1989).

[4] J. A. Bondy and U. S. R. Murty, Graph theory, Springer, (2008).

[5] A. Brandstädt, V. B. Le and J. P. Spinrad, Graph classes: A survey, SIAM, Philadelphia, (1999).

[6] G. Chartrand and P. Zhang, Introduction to graph theory, McGrawHill Inc., (2005).

[7] N. Deo, Graph theory with applications to engineering and computer science, PHI Learning, Delhi, (1974).

[8] J. A. Gallian, A dynamic survey of graph labeling, Electron. J. Combin. (DS-6), (2014).

[9] K. A. Germina and T. M. K. Anandavally, Integer additive set-indexers of a graph: sum square graphs, J. Combin. Inform. System Sci., 37(24), pp. 345-358, (2012). 
[10] K. A. Germina and N. K. Sudev, On weakly uniform integer additive set-indexers of graphs, Int. Math. Forum., 8(37)(2013), 1827-1834. DOI: 10.12988/imf.2013.310188.

[11] J. Gross and J. Yellen, Graph theory and its applications, CRC Press, (1999).

[12] F. Harary, Graph theory, Addison-Wesley Pub. Co. Inc., (1969).

[13] K. D. Joshi, Applied discrete structures, New Age International, (2003).

[14] S. M. Hegde, Numbered graphs and their applications, Ph.D. Thesis, Delhi University, India, (1989).

[15] M. B. Nathanson Additive number theory: Inverse problems and geometry of sumsets, Springer, (1996).

[16] N. K. Sudev and K. A. Germina, On integer additive set-indexers of graphs, Int. J. Math. Sci.\& Engg. Appl., 8(2), pp. 11-22, (2014).

[17] N. K. Sudev and K. A. Germina, Some new results on strong integer additive set-indexers of graphs, Discrete Math. Algorithms Appl., 7(1)(2015), 1-11., DOI: 10.1142/S1793830914500657.

[18] N. K. Sudev and K. A. Germina, A study on arithmetic integer additive set-indexers of graphs, Carpathian Math. Pub., J. Inform. Math. Sci.

[19] N. K. Sudev and K. A. Germina, On certain arithmetic integer additive set-indexers of graphs, Discrete Math. Algorithms Appl., 7(3)(2015), 1-15., DOI: 10.1142/S1793830915500251.

[20] N. K. Sudev and K. A. Germina, A study on semi-arithmetic integer additive set-indexers of graphs, Int. J. Math. Sci. \& Engg. Appl., 8(3), pp. 157-165, (2014).

[21] D. B. West, Introduction to graph theory, Pearson Edu. Inc., (2001). 


\section{N. K. Sudev}

Centre for Studies in Discrete Mathematics

Vidya Academy of Science \& Technology

Thrissur - 680501, Kerala,

India

e-mail : sudevnk@gmail.com

and

\section{K. A. Germina}

Department Mathematics

Central University of Kerala

Periya, Kasaragod,

India

e-mail : srgerminaka@gmail.com 